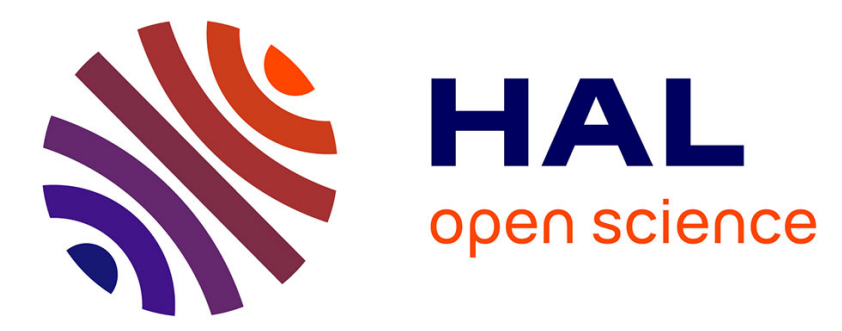

\title{
Control of Discrete Event Systems with Respect to Strict Duration: Supervision of an Industrial Manufacturing Plant
}

\author{
Abdourrahmane Atto, Claude Martinez, Said Amari
}

\section{To cite this version:}

Abdourrahmane Atto, Claude Martinez, Said Amari. Control of Discrete Event Systems with Respect to Strict Duration: Supervision of an Industrial Manufacturing Plant. Computers \& Industrial Engineering, 2011, 61 (4), pp.Pages 1149-1159. 10.1016/j.cie.2011.07.004 hal-00660726

\section{HAL Id: hal-00660726 https://hal.science/hal-00660726}

Submitted on 17 Jan 2012

HAL is a multi-disciplinary open access archive for the deposit and dissemination of scientific research documents, whether they are published or not. The documents may come from teaching and research institutions in France or abroad, or from public or private research centers.
L'archive ouverte pluridisciplinaire HAL, est destinée au dépôt et à la diffusion de documents scientifiques de niveau recherche, publiés ou non, émanant des établissements d'enseignement et de recherche français ou étrangers, des laboratoires publics ou privés. 


\title{
Control of Discrete Event Systems with Respect to Strict Duration: Supervision of an Industrial Manufacturing Plant
}

\author{
Abdourrahmane M. ATTO * Claude MARTINEZ ${ }^{\dagger}$ \\ Saïd AMARI ‡
}

\begin{abstract}
In this paper, we propose a $(\max ,+)$-based method for the supervision of discrete event systems subject to tight time constraints. Systems under consideration are those modelled as timed event graphs and represented with linear $(\max ,+)$ state equations. The supervision is addressed by looking for solutions of constrained state equations associated with timed event graph models. These constrained state equations are derived by reducing duration constraints to elementary constraints whose contributions are injected in the system's state equations. An example for supervisor synthesis is given for an industrial manufacturing plant subject to a strict temporal constraint, the thermal treatment of rubber parts for the automotive industries. Supervisors are calculated and classified according to their performance, considering their impact on the production throughput.
\end{abstract}

Keywords: Discrete event systems, $(\max ,+)$ algebra, dioids, time constraints, supervisor, control.

\section{Introduction}

Discrete Event Systems (DES) are of great interest in research activities dedicated to industrial production systems. Many approaches have been proposed for the analysis of DES these last few decades (see [6] among others). Some are related to computational simulations [22], and others are based on the (max, +)algebra $[4,14]$. Under some assumptions, DES can be modelled as Timed Event Graphs (TEGs) $[14,25]$ and thus, the analysis of the system can be described with linear equations in $(\max ,+)$-algebra.

This work concerns the supervision of DES, modelled as TEGs, and assumed to respect strict temporal constraints for specific processing. The supervision is aimed at guaranteeing the respect of temporal constraints without impacting significantly the dynamic behaviour of the system. Similar problems of

\footnotetext{
*LISTIC, Université de Savoie, Polytech Annecy-Chambry, France, am.atto@ieee.org

${ }^{\dagger}$ IRCCyN UMR CNRS 6597, LUNAM Université, Université de Nantes, Nantes, France, claude.martinez@univ-nantes.fr

${ }^{\ddagger}$ LURPA, ENS Cachan, France, said.amari@lurpa.ens-cachan.fr
} 
meeting time constraints have been recently addressed via different approaches $[27,21,3,26,20]$. We propose solutions based on a constrained $(\max ,+)$ state equation for the TEG model of the DES. Constrained state equations are obtained by reducing temporal constraints to elementary constraints and by injecting contributions of these elementary constraints in the state equation of the TEG models. These elementary constraint equations derive from a simplified representation of the TEG under consideration, representation which consists in decomposing a place with a number $m$ of tokens into $m$ places, each one containing only one token.

The method proposed in this paper is used to synthesize supervisors for an industrial manufacturing plant subject to a strict temporal constraint. Supervisors are calculated and classified according to their performance. The performance evaluation is of great interest in the literature on the topic of $(\max ,+)$ algebra $[4,14,11]$. The performance of a supervisor is measured according to the maximum throughput of the plant. According to this particular performance measure, we can classify the supervisors between those which slow down the production throughput and those which preserve this production rate. The cycle time of such a plant modelled as a TEG corresponds to the eigenvalue of the matrix associated with its graph $[4,9,15]$; the production throughput is the inverse of the cycle time.

This work is organised as follows. Section 2 provides basics on $(\max ,+)$ theory. Then a description is given for the dynamic behaviour of TEGs according to linear $(\max ,+)$ models (section 3$)$, and the integration of temporal constraints is discussed for this linear model. Section 4 addresses the supervision problem and provides a method to synthesise supervisors. A first example is given for the supervision of a constrained system. Section 5 illustrates the proposed method for the control a single armed robot in a cluster-tool for the semiconductor industry. Section 6 discusses the supervision of an industrial plant. The supervision is aimed at guaranteeing the respect of a strict duration constraint for a heating process without impacting significantly the production rate of the manufacturing unit. In section 6 , we show that the supervision can be performed thanks to the analytical technique described in section 4 and provide supervisors that allow for preserving the production rate of the industrial plant. Finally, section 7 gives a conclusion and addresses perspectives to extend this work.

\section{$2(\max ,+)$ algebra}

This section briefly recalls the fundamentals of $(\max ,+)$ algebra, which is largely used for the analysis of DES. Further details about this theory can be found in $[16,10,14,8,23]$ among others. Some specific results that are essentials to state on the existence of a solution to the problem tackled here are therefore given at the end of the section. In what follows, $\mathcal{D}$ denotes a set.

Definition 1 (Monoid) A monoid is an algebraic set with an associative internal operation and an identity element.

Definition 2 (Semiring) $(\mathcal{D}, \oplus, \otimes)$ is a semiring if:

- $(\mathcal{D}, \oplus)$ is a commutative monoid. Its identity element is denoted by $\epsilon$ (null element). 
- $(\mathcal{D}, \otimes)$ is a monoid. Its identity element is denoted by e (unit element).

- Multiplication $\otimes$ distributes over addition and $\epsilon$ annihilates $\mathcal{D}$ (every $x \in$ $\mathcal{D}$ is such that $x \otimes \epsilon=\epsilon \otimes x=\epsilon$ ).

Definition 3 (Dioid) $A$ dioid $(\mathcal{D}, \oplus, \otimes)$ is an idempotent semiring (every $x \in$ $\mathcal{D}$ is such that $x \oplus x=x)$.

Hereafter, the product $a \otimes b$ will be denoted $a . b$ or $a b$ when there is no possible confusion.

\section{Example 1 (Examples of dioids)}

- Let $\mathbb{R}$ be the set of real numbers. $(\mathbb{R} \cup\{-\infty\}$, $\max ,+)$ is a commutative dioid for which $\epsilon=-\infty$ and $e=0$. This dioid is denoted by $\mathbb{R}_{\max }$ and is called $(\max ,+)$ algebra.

- Let $(\mathcal{D}, \oplus, \otimes)$ be a dioid and $\mathcal{D}^{n \times n}$ the set of square matrices of order $n$ over $\mathcal{D}$. $\left(\mathcal{D}^{n \times n}, \oplus, \otimes\right)$ is a dioid called a matrix dioid. The sum and the matrix product are defined as follows: if $\boldsymbol{A}=\left(A_{i j}\right), \boldsymbol{B}=\left(B_{i j}\right)$, then $(\boldsymbol{A} \oplus \boldsymbol{B})_{i j}=A_{i j} \oplus B_{i j}$ and $(\boldsymbol{A} \otimes \boldsymbol{B})_{i j}=\bigoplus_{k=1}^{n} A_{i k} \otimes B_{k j}$. The null element of the matrix dioid is the matrix composed of $\epsilon$. The unit matrix is the matrix with $e$ on the main diagonal and $\epsilon$ elsewhere.

Definition 4 (Moduloid) Let $\mathcal{D}$ be a dioid. $A$ moduloid $\mathcal{M}$ over $\mathcal{D}$ is a monoid $(\mathcal{M}, \oplus)$, endowed with an external operation "." $\mathcal{D} \times \mathcal{M} \rightarrow \mathcal{M}$, such that for all $\lambda, \mu \in \mathcal{D}$ and for all $u, v \in \mathcal{M}$ :

1. $(\lambda \oplus \mu) \cdot u=\lambda \cdot u \oplus \mu \cdot v$

2. $(\lambda . \mu) \cdot u=\lambda \cdot(\mu . u)$

3. $\lambda(u \oplus v)=\lambda . u \oplus \lambda . v$

4. $\epsilon \cdot u=\epsilon$

5. e. $u=u$

Example 2 (Examples of moduloids) The set $\mathcal{D}^{n}$ of $n$ dimensional vectors over a dioid $\mathcal{D}$ is a moduloid over $\mathcal{D}$. In the same way, the set of $\mathcal{D}^{n \times m}$ matrices over a dioid $\mathcal{D}$ is a moduloid over $\mathcal{D}$.

In the rest of the paper (especially in section 4), we consider the moduloid $\mathbb{R}_{\max }^{n \times m}$ defined over the dioid $\mathbb{R}_{\max }$.

Let $(\mathcal{D}, \oplus, \otimes)$ be a dioid. The idempotency of the operation $\oplus$ induces over $\mathcal{D}$, an order structure denoted $\preceq$ and defined by: $x \preceq y \Leftrightarrow x \oplus y=y$. This order relation is compatible with the operations $\oplus$ and $\otimes($ proof in [4]). In $(\max ,+)$ algebra, this order coincides with usual order $\leqslant$. The lower $(\wedge)$ and upper $(\vee)$ bounds are defined by: $x \preceq y \Leftrightarrow x \oplus y=y \Leftrightarrow x \wedge y=x \Leftrightarrow x \vee y=y=x \oplus y$. 
Definition 5 (Completeness in dioids) $A$ dioid $(\mathcal{D}, \oplus, \otimes)$ is complete if

$$
\forall c \in \mathcal{D}, \forall A \subseteq \mathcal{D}, c \otimes\left(\bigoplus_{x \in A} x\right)=\bigoplus_{x \in A} c \otimes x
$$

that is if it is closed for infinite sums and if the operation $\otimes$ distributes over infinite sums.

A complete dioid $\mathcal{D}$ is upper bounded by an element, denoted $T$, defined by:

$$
T=\bigoplus_{x \in \mathcal{D}} x
$$

This element annihilates $(\mathcal{D}, \oplus)$, that is $T \oplus x=T$ for all $x \in \mathcal{D}$, and this element also verifies: $T \otimes \epsilon=\epsilon$.

Note that dioid $\mathbb{R}_{\max }$ is not complete. $\mathbb{R}_{\max } \cup\{+\infty\}$ with the convention: $(-\infty) \otimes(+\infty)=(+\infty) \otimes(-\infty)=-\infty$ is a complete dioid denoted by $\overline{\mathbb{R}}_{\max }$.

Theorem 1 Let $\mathcal{D}$ be a complete dioid. The least solution in $x$ of $x \succeq a x \oplus b$ is $x=a^{*} b$, where $a^{*}=\bigoplus_{k \geq 0} a^{k}$ is the Kleene star of $a$.

The proof of this theorem is given in [14].

The problem of multivariable control tackled in this article involves solving an inequality of the form $\boldsymbol{A} \otimes \boldsymbol{x} \geqslant \boldsymbol{B} \otimes \boldsymbol{x}$. In this problem, it is often usefull to address the existence of solutions that makes synthesis of controller possible. In this respect, the following lemma is actually a specific case of the results presented in Allamigeon et al [1]. Earlier works on resolution of this type of inequality can be found in Butkovič et al [17, 13, 5], in [28] from Walkup et al and in Cechlárová [7].

Lemma 1 Let $\boldsymbol{v}, \boldsymbol{u} \in \mathbb{R}_{\max }^{1 \times n}$ be given row vectors and $\boldsymbol{x} \in \mathbb{R}_{\max }^{n}$. The inequality $\boldsymbol{v} \otimes \boldsymbol{x} \geqslant \boldsymbol{u} \otimes \boldsymbol{x}$ admits a non-trivial solution if the following necessary and sufficient condition holds: $\exists k \in \bar{n} \mid v_{k} \geqslant u_{k}$.

Let us call such index $k$ a critical index. Let $K_{i}$ be the set containing all critical indices in a row $i$ of a matrix $\boldsymbol{H}$. Cechlárová gives in [7] a necessary and sufficient condition for the existence of a solution for equation of the form $\boldsymbol{H} \otimes \boldsymbol{x}=\boldsymbol{Q} \otimes \boldsymbol{x}$ with $\boldsymbol{H} \geqslant \boldsymbol{Q}$. This condition is expressed in the following theorem. The proof of this theorem is given in [7]. In this theorem, $\bar{p}$ denotes the set of row indices $\{1,2, \ldots, p\}$ and $\bar{n}$ is the set of column indices $\{1,2, \ldots, m\}$ of matrices $\boldsymbol{H}$ and $\boldsymbol{Q}$.

Theorem 2 A system $\boldsymbol{H} \otimes \boldsymbol{x}=\boldsymbol{Q} \otimes \boldsymbol{x}$ with $\boldsymbol{H} \in \overline{\mathbb{R}}_{\max }^{p \times n}, \boldsymbol{H} \geqslant \boldsymbol{Q}$, is soluble if and only if

1. each $K_{i}$ is nonempty,

2. there exist a function $j: \bar{p} \rightarrow \bar{n}$, with $j(i) \in K_{i}$ for all $i \in \bar{p}$ and satisfying: for every subset $\left\{i_{1}, i_{2}, \ldots, i_{k}\right\}$ of $\bar{p}$ such that the indices $j\left(i_{1}\right), j\left(i_{2}\right), \ldots, j\left(i_{k}\right)$ are pairwise different, then we have,

$$
h_{i_{1} j\left(i_{1}\right)}+h_{i_{2} j\left(i_{2}\right)}+\cdots+h_{i_{k} j\left(i_{k}\right)} \geqslant h_{i_{1} j\left(i_{2}\right)}+h_{i_{2} j\left(i_{3}\right)}+\cdots+h_{i_{k} j\left(i_{1}\right)} .
$$




\section{TEG and linear $(\max ,+)$ models}

This section recalls modelling tools for describing the dynamic behaviour of TEGs (in subsection 3.1) and provides a natural way of integrating the time constraint in TEGs (subsection 3.2).

\subsection{Dynamic behaviour}

A Petri net consists of places, directed arcs, and transitions. Directed arcs connect places and transitions (there is no direct connection between two places or between two transitions). TEGs are a subclass of Petri Nets in which every place is connected to only one input and one output transition. According to the nature of the problem tackled in this paper, we focus on the particular case where crossing transitions is instantaneous. In such cases, temporisations are set only over places [25]. The temporisation associated with each place corresponds to the minimum duration of a specific process running in this place and marked by a token. A place with temporisation $e=0.0$ is simply represented without its temporisation. Each transition $x_{j}$ is associated with a function $x_{j}(k)$ that gives the earlier firing time for the $k^{\text {th }}$ occurrence of event $j$. An example of a TEG is given in figure 1. The dynamic behaviour of this graph is described by

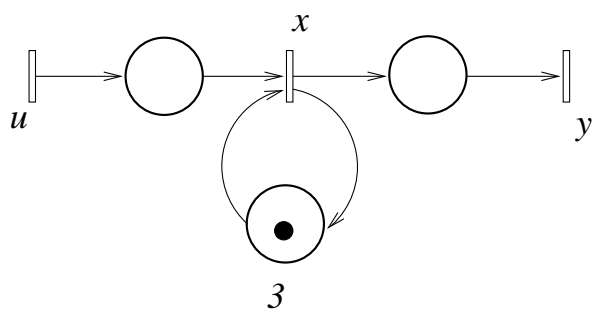

Figure 1: TEG example.

the system (firing times)

$$
\left\{\begin{array}{l}
x(k+1)=3 x(k) \oplus u(k+1) \\
y(k)=x(k)
\end{array}\right.
$$

A TEG being a directed graph, its dynamic behaviour can be described thanks to matrices associated with connected components of the graph. Generally, this dynamic behaviour is represented by a system:

$$
\left\{\begin{array}{l}
\boldsymbol{x}(k+1)=\boldsymbol{A} \boldsymbol{x}(k) \oplus \boldsymbol{B} \boldsymbol{v}(k+1), \\
\boldsymbol{y}(k)=\boldsymbol{C} \boldsymbol{x}(k),
\end{array}\right.
$$

where $\boldsymbol{v}$ denotes the input vector, $\boldsymbol{x}$ the state vector, and $\boldsymbol{y}$ the output vector. The first equation of (1) is the state equation (all TEGs can be modeled by a state equation of this form [18]), while the second one is the output equation of the TEG. For a TEG featuring $N$ transitions, given an initial vector $\boldsymbol{x}_{0}$ the set $\left\{x_{i}(k) \mid 1 \leqslant i \leqslant N: k=1,2, \ldots, K\right\}$ denotes a firing schedule [21]. 


\subsection{Maximal duration constraints}

On one hand, the minimum duration of tokens in places is expressed by temporisations of these places. On the other hand, if we wish to express a maximum duration in a place, we must then add an additional constraint.

A maximum duration constraint is defined as a critical time that should not be exceeded for a given process. Let $\tau_{\max }$ be a maximum duration constraint for a certain process and $\tau$ be the normal duration of this process. We have $\tau_{\max } \geqslant \tau$ since otherwise, the process will be stopped before being fully completed. Thus maximum constraints consist in setting upper-bounds in order to avoid, for sensitive processes, too long or even excessive processing times which can occur when strong disturbances occur in the system.

Note that the state equation of a TEG involves a representation where each place contains either zero or one token (any TEG can be modeled by a state equation of the form Eq. (1), see [18] among others). Thus, a set of constraints can also be decomposed so as for the contraints to apply on the simplified representation of the TEG. In this sense, a set of constraints can be expressed as a function of $\boldsymbol{x}(k+1)$ and $\boldsymbol{x}(k)$. In what follows, we are interested in contraints that have the following form:

$$
\boldsymbol{C}_{0} \boldsymbol{x}(k+1) \oplus \boldsymbol{C}_{1} \boldsymbol{x}(k) \leqslant \boldsymbol{C}_{\max } \boldsymbol{x}(k+1),
$$

where $\boldsymbol{C}_{\max }$ contains a set of critical durations that must not be exceeded and $\boldsymbol{C}_{0}, \boldsymbol{C}_{1}$ describe how these maximal duration constrain the TEG.

Let us consider the TEG represented in figure 2.

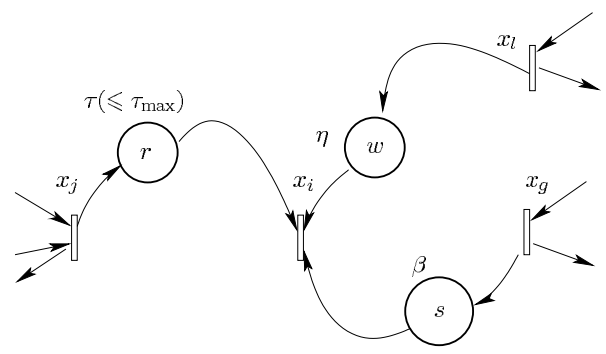

Figure 2: Temporal constraint

Let $p_{i j}$ be the place linking transition $x_{j}$ to transition $x_{i}$. The marking (number of tokens) of place $p_{i j}$ is $r$. If we want to enforce a maximum time duration $\tau_{\max }$ (with $\tau_{\max } \geqslant \tau$ ) to tokens in place $p_{i j}$, then the following inequality must be satisfied:

$$
x_{i}(k) \leqslant \tau_{\max } x_{j}(k-r) .
$$

In addition, according to the graph of figure 2 , transition $x_{i}$ firing is governed by

$$
x_{i}(k)=\tau x_{j}(k-r) \oplus \beta x_{g}(k-s) \oplus \eta x_{l}(k-w) .
$$

The above Eqs. (3) and (4) can be rewritten in terms of $\boldsymbol{x}(k+1)$ and $\boldsymbol{x}(k)$ by decomposing any place with more than one token in places having only one token. Such a decomposition have been performed in the application given by Section 6.1. Concerning this example, we simply consider the special case where $r=0$ and $s=w=1$. By taking into account Eqs. (3) and (4), we derive the 
(necessary and sufficient) condition under which a token will not exceed the duration constraint $\tau_{\max }$ in place $p_{i j}$ :

$$
\beta x_{g}(k) \oplus \eta x_{l}(k) \leqslant \tau_{\max } x_{j}(k+1) .
$$

This constraint is of the form: $\boldsymbol{C}_{0} \boldsymbol{x}(k+1) \oplus \boldsymbol{C}_{1} \boldsymbol{x}(k) \leqslant \boldsymbol{C}_{\max } \boldsymbol{x}(k+1)$, with

$$
\begin{aligned}
& \boldsymbol{x}=\left(\begin{array}{llll}
x_{g} & x_{i} & x_{j} & x_{l}
\end{array}\right)^{\prime}, \\
& \boldsymbol{C}_{0}=\left(\begin{array}{llll}
\epsilon & \epsilon & \tau & \eta
\end{array}\right), \quad \boldsymbol{C}_{1}=\left(\begin{array}{llll}
\beta & \epsilon & \epsilon & \epsilon
\end{array}\right), \\
& \boldsymbol{C}_{\max }=\left(\begin{array}{llll}
\epsilon & \epsilon & \tau_{\max } & \epsilon
\end{array}\right),
\end{aligned}
$$

and $\ll^{\prime} \gg$ denoting matrix transposition. Note that $\boldsymbol{C}_{0}$ and $\boldsymbol{C}_{1}$ are obtained from matrices $\boldsymbol{A}_{0}$ and $\boldsymbol{A}_{1}$ and $\boldsymbol{C}_{\text {max }}$ depends on the maximum time duration. The next example involves two duration constraints.

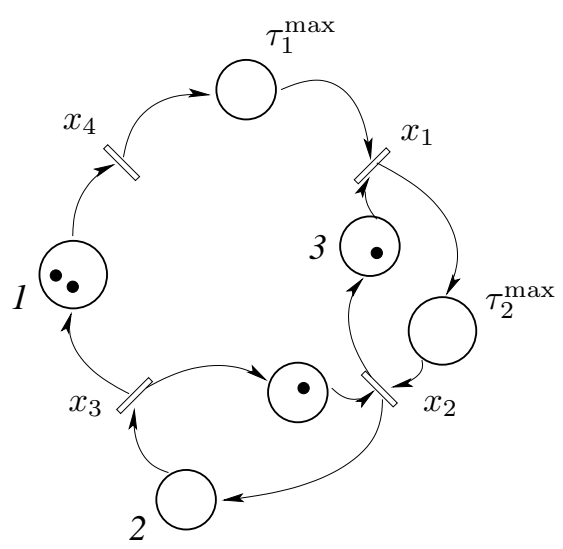

Figure 3: TEG with two duration constraints.

Example 3 Consider the TEG given in figure 3. Durations $\tau_{1}=\tau_{1}^{\max }$ and $\tau_{2}=\tau_{2}^{\max }$ are the normal duration of tokens in corresponding places and these durations should not be exceeded. The constraints are:

$$
\begin{aligned}
& \text { (a) } x_{1}(k) \leqslant \tau_{1}^{\max } x_{4}(k) \text {, } \\
& \text { and } \\
& \text { (b) } x_{2}(k) \leqslant \tau_{2}^{\max } x_{1}(k) .
\end{aligned}
$$

These constraints are respected if:

$$
\begin{array}{ll}
\text { (a) true if } & 3 x_{2}(k-1) \leqslant \tau_{1}^{\max } x_{4}(k), \\
\text { (b) true if } & x_{3}(k-1) \leqslant \tau_{2}^{\max } x_{1}(k),
\end{array}
$$

that is, if

$$
\left(\begin{array}{cccc}
\tau_{2} & \epsilon & e & \epsilon \\
\epsilon & 3 & \epsilon & \tau_{1}
\end{array}\right) \boldsymbol{x}(k) \leqslant\left(\begin{array}{cccc}
\tau_{2}^{\max } & \epsilon & \epsilon & \epsilon \\
\epsilon & \epsilon & \epsilon & \tau_{1}^{\max }
\end{array}\right) \boldsymbol{x}(k+1),
$$

with $\boldsymbol{x}=\left(x_{1} x_{2} x_{3} x_{4}\right)^{\prime}$. 
A set of constraint equations does not always admit a solution that makes supervisor synthesis possible. Indeed, the set of state equation and constraint equation can lead to an ill posed problem. In this respect, we will say that a set of maximum duration constraints fits the normal behaviour (described by the state equation) of a TEG if the set of state and constraint equations admit a solution. We will also say that the firing schedule $\left\{x_{i}(k) \mid 1 \leqslant i \leqslant N: k=1,2, \ldots, K\right\}$ is consistant if all constraints are satisfied. As an example, imposing $\tau_{\max }<8$ (resp. $\tau_{\max }<3$ ) for the TEG \# 1 (resp. TEG \# 2) of figure 4 does not fit the normal behaviour of the system since the normal duration of the process (resp. the synchronization) involved in TEG \# 1 (resp. TEG \# 2) is greater than or equal to 8 (resp. 3) time units. Therefore no consistant schedule exist, given these constraints on those TEG.
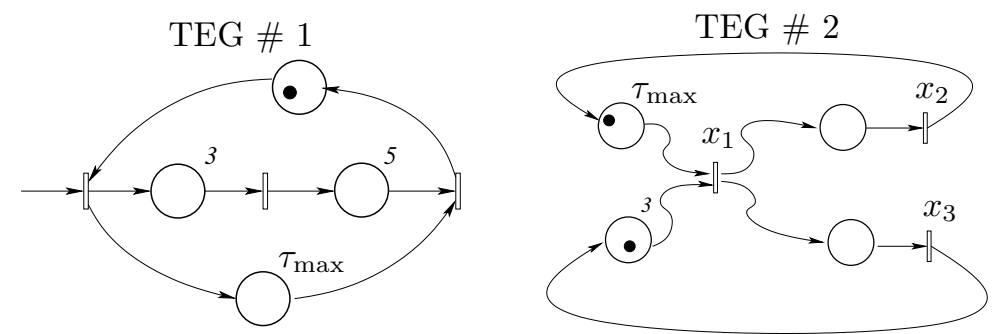

Figure 4: Imposing a constraint $\tau_{\max }<8$ (resp. $\tau_{\max }<3$ ) for TEG \# 1 (resp. TEG \# 2) does not fit the normal behaviour of the system since the TEG state equations impose a processing time $\tau_{\max } \geqslant 8$ (resp. $\tau_{\max } \geqslant 3$ ) for TEG \# 1 (resp. TEG \# 2).

\section{Supervisor synthesis}

This section provides a method for synthesising supervisors of TEGs subject to a maximum temporal constraint. We assume that transitions of the TEG are all controllable, that is, firing of each transition can be disabled by a supervisor. We propose to add a supervisor for the TEG in order to guarantee respect of the duration constraint for every input $v$. The supervision is justified by the following result.

Theorem 3 Consider a linear $(\max ,+)$ system governed by the state equation

$$
\boldsymbol{x}(k+1)=\boldsymbol{A} \boldsymbol{x}(k) \oplus \boldsymbol{B} \boldsymbol{v}(k+1),
$$

where $\boldsymbol{A}$ and $\boldsymbol{B}$ are matrices with dimensions $n \times n$ and $n \times m, n$ and $m$ being the number of state transitions and the number of input transitions respectively.

Assume that this system is subject to maximum duration constraints:

$$
\boldsymbol{C}_{0} \boldsymbol{x}(k+1) \oplus \boldsymbol{C}_{1} \boldsymbol{x}(k) \leqslant \boldsymbol{C}_{2} \boldsymbol{x}(k+1),
$$

where $\boldsymbol{C}_{0}, \boldsymbol{C}_{1}$ and $\boldsymbol{C}_{2}$ are matrices with dimensions $\ell \times n$, $\ell$ being the number of constraints. 
A controller that allows the duration constraints to be satisfied can be obtained by modifying the state equation in the form (constrained state equation):

$$
\boldsymbol{x}(k+1)=(\boldsymbol{A} \oplus \boldsymbol{M}) \boldsymbol{x}(k) \oplus \boldsymbol{B} \boldsymbol{v}(k+1),
$$

via a modification matrix $\boldsymbol{M}$, which is such that:

$$
\boldsymbol{C}_{0}(\boldsymbol{A} \oplus \boldsymbol{M}) \oplus \boldsymbol{C}_{1} \leqslant \boldsymbol{C}_{2} \boldsymbol{M} .
$$

Proof : If $\boldsymbol{x}(k+1)=\boldsymbol{A} \boldsymbol{x}(k) \oplus \boldsymbol{M} \boldsymbol{x}(k) \oplus \boldsymbol{B} \boldsymbol{v}(k+1)$, then

$$
\boldsymbol{C}_{2} \boldsymbol{x}(k+1)=\boldsymbol{C}_{2} \boldsymbol{A} \boldsymbol{x}(k) \oplus \boldsymbol{C}_{2} \boldsymbol{M} \boldsymbol{x}(k) \oplus \boldsymbol{C}_{2} \boldsymbol{B} \boldsymbol{v}(k+1) .
$$

If : $\boldsymbol{C}_{1} \leqslant \boldsymbol{C}_{2} \boldsymbol{M}$, then

$$
\boldsymbol{C}_{2} \boldsymbol{x}(k+1) \geqslant \boldsymbol{C}_{2} \boldsymbol{A} \boldsymbol{x}(k) \oplus \boldsymbol{C}_{1} \boldsymbol{x}(k) \oplus \boldsymbol{C}_{2} \boldsymbol{B} \boldsymbol{v}(k+1) .
$$

Thus,

$$
\boldsymbol{C}_{1} \boldsymbol{x}(k) \leqslant \boldsymbol{C}_{2} \boldsymbol{x}(k+1) .
$$

And if : $\boldsymbol{C}_{0}(\boldsymbol{A} \oplus \boldsymbol{M}) \leqslant \boldsymbol{C}_{2} \boldsymbol{M}$, then

$$
\boldsymbol{C}_{2} \boldsymbol{x}(k+1) \geqslant \boldsymbol{C}_{2} \boldsymbol{A} \boldsymbol{x}(k) \oplus \boldsymbol{C}_{0}(\boldsymbol{A} \oplus \boldsymbol{M}) \boldsymbol{x}(k) \oplus \boldsymbol{C}_{2} \boldsymbol{B} \boldsymbol{v}(k+1) .
$$

Thus,

$$
\boldsymbol{C}_{0}(\boldsymbol{A} \oplus \boldsymbol{M}) \boldsymbol{x}(k) \leqslant \boldsymbol{C}_{2} \boldsymbol{x}(k+1),
$$

and the result follows.

Remark 1 We have:

- Supervisors guaranteeing respect of the duration constraints correspond to $\boldsymbol{M}$-solutions (when they exist) of the inequality $\boldsymbol{C}_{0}(\boldsymbol{A} \oplus \boldsymbol{M}) \oplus \boldsymbol{C}_{1} \leqslant$ $\boldsymbol{C}_{2} \boldsymbol{M}$. Note that in the case where $\boldsymbol{C}_{0} \boldsymbol{A} \oplus \boldsymbol{C}_{1} \leqslant \boldsymbol{C}_{2} \boldsymbol{A}$, the maximal duration constraints are satisfied without any supervision.

- As mentioned in section 3.2 above, $\boldsymbol{C}_{0}$ and $\boldsymbol{C}_{1}$ still depend on matrices $\boldsymbol{A}_{0}$ and $\boldsymbol{A}_{1}$ whereas $\boldsymbol{C}_{2}$ depends only on maximum time durations.

- The existence of a modification matrix $\boldsymbol{M}$ depends on the constraints we want to impose. For a single constraint, $\ell=1$, equation 7 reduces to an inequality of the form $\boldsymbol{u} \otimes \boldsymbol{y} \oplus y_{0} \leqslant \boldsymbol{v} \otimes \boldsymbol{y}$ for each column of $\boldsymbol{M}$ and Lemma 1 can be applied. For a set of constraints $(\ell \geqslant 2)$, Theorem 2, applied to the columns of $\boldsymbol{M}$, makes it possible to seek for the existence of solutions in $\boldsymbol{M}$ for the constrained system.

Example 4 By considering again Example 3, condition given by Eq. (9) reduces to $\boldsymbol{C}_{1} \leqslant \boldsymbol{C}_{2} \boldsymbol{M}$ and a modification matrix $\boldsymbol{M}$ can easily be determined. Indeed, we are concerned with the two conditions:

$$
e \leqslant \tau_{2}^{\max } m_{1,3}, \quad \text { and } \quad 3 \leqslant \tau_{1}^{\max } m_{4,2} .
$$

A corresponding supervisor is thus a set of two places $\left\{p_{1,3}, p_{4,2}\right\}$, each one containing one token. The temporisations of the places are: null $(e)$ for place $p_{1,3}$ and $\left(3-\tau_{1}^{\max } \oplus e\right)$ for place $p_{4,2}$. The graph of figure 3 , provided with this supervisor is represented in figure 5 . 


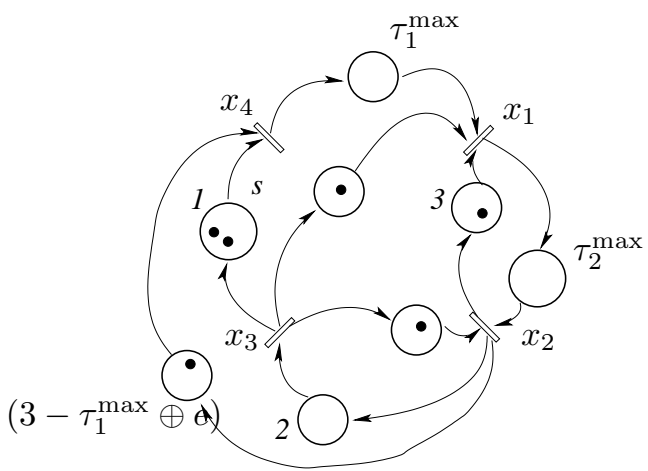

Figure 5: Supervision of the TEG given in figure 3, with respect to the strict duration constraints $\tau_{1}^{\max }$ and $\tau_{2}^{\max }$.

\section{Example: control of robots in cluster-tools}

The scheduling a control of robots in cluster-tools dedicated to the semiconductor industry has been deeply studied in the last decade [21, 29]. The TEG considered in this example, see Figure 6, is a simplifyed instance of a model given by Wu et al [29] that represents the behaviour of a single armed robot in a $(2,1)$ schedule of a cluster-tool. Briefly, a cluster-tool dedicated to semiconductor industry is a set of processing modules (PM) where a chemeical or a thermal treatment is applied to a waffer. A robot unloads the waffers one by one and aligns them from a load-lock and serves the different PM. The robot also unloads the waffer from a PM and transports it to the next PM or to the load-lock when the complete process has completed. A waffer is generally processed in several steps. In this example, the cluster-tool has three PMs, two are dedicated to the first step, and the third to the second step. The durations of these elementary tasks are: unloading from the load-lock and aligning a waffer, $\lambda_{0}$ time units, unloading from a PM or loadind to a PM or to the load-lock (no aligning is necessary), $\lambda$ time units, a robot move, $\mu$ time units, process step one, $\tau_{1}$, process step two, $\tau_{2}$. Additional durations $d_{1}$ and $d_{2}$ are tolerated to step one and two.

The dynamic behaviour of this TEG is governed by $\boldsymbol{x}(k+1)=\mathbf{A}_{\mathbf{0}}{ }^{*} \mathbf{A}_{\mathbf{1}} \boldsymbol{x}(k)$, and duration constraints are given by $\boldsymbol{C}_{0} \boldsymbol{x}(k+1) \leqslant \boldsymbol{C}_{2} \boldsymbol{x}(k+1)$, with the following matrices where $\epsilon$ has again been replaced by a dot,

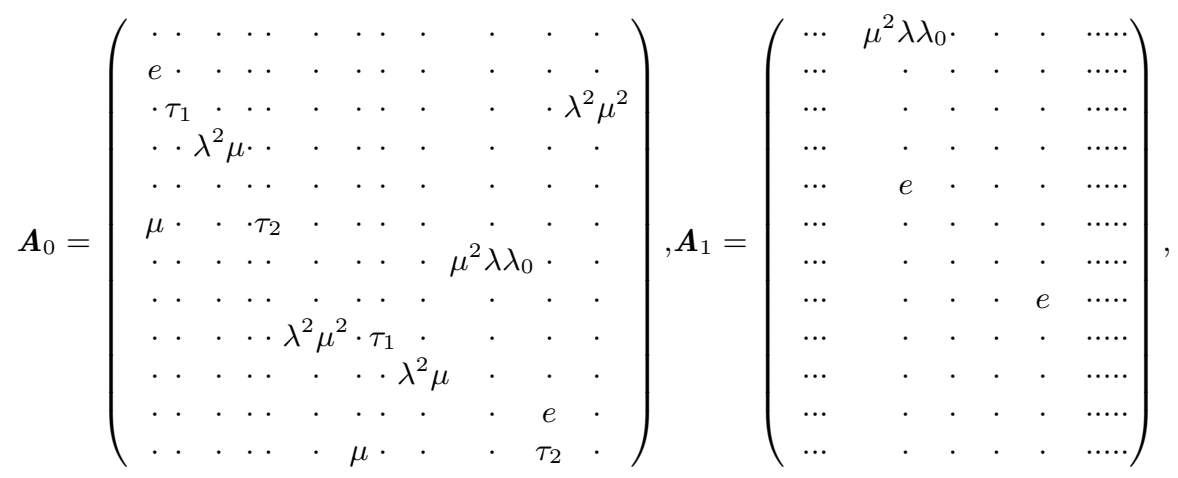




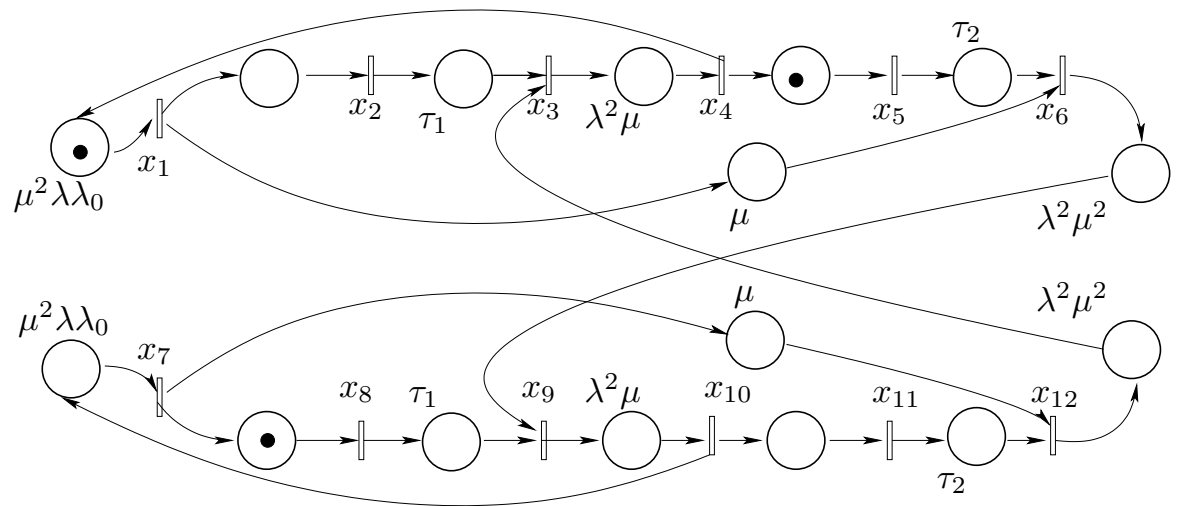

Figure 6: A model of a single armed cluster-tool in a $(2,1)$ schedule (Supervision discussed in Section 5), the strict duration constraints are $\tau_{1}^{\max }=\tau_{1} d_{1}$ and $\tau_{2}^{\max }=\tau_{2} d_{2}$.

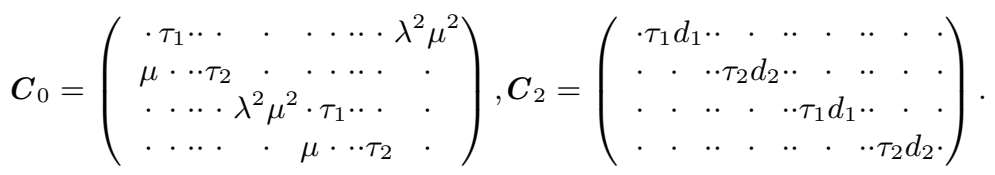

The above constraint can be written in the form $\boldsymbol{C}_{0} A_{0}^{*} A_{1} \oplus \boldsymbol{C}_{0} \boldsymbol{M} \leqslant \boldsymbol{C}_{2} \boldsymbol{M}$, which is also equivalent to the system of inequalities:

$$
\left\{\begin{array}{lcc}
C_{0} A_{0}^{*} A_{1} & \leqslant C_{2} M \\
C_{0} M & \text { and } & \leqslant C_{2} M .
\end{array}\right.
$$

Let us check for the existence of solutions in $\boldsymbol{M}$ that satisfies inequality $\boldsymbol{C}_{0} \boldsymbol{M} \leqslant \boldsymbol{C}_{2} \boldsymbol{M}$. This can be performed either by using Theorem 2 or by trying to solve directly, the inequalities involves.

- Method 1 : from Theorem 2.

We have that $\boldsymbol{C}_{0} \boldsymbol{M} \leqslant \boldsymbol{C}_{2} \boldsymbol{M}$ is equivalent to $\boldsymbol{C}_{0} \boldsymbol{M} \oplus \boldsymbol{C}_{2} \boldsymbol{M} \leqslant \boldsymbol{C}_{2} \boldsymbol{M}$, that is, to $\left(\boldsymbol{C}_{0} \oplus \boldsymbol{C}_{2}\right) \boldsymbol{M} \leqslant \boldsymbol{C}_{2} \boldsymbol{M}$. Let us consider the equation $\left(\boldsymbol{C}_{0} \oplus \boldsymbol{C}_{2}\right) \boldsymbol{M}=$ $\boldsymbol{C}_{2} \boldsymbol{M}$. One can seek for a solution to this equation by checking the conditions of Theorem 2, with $\boldsymbol{H}=\boldsymbol{C}_{0} \oplus \boldsymbol{C}_{2}$ and $\boldsymbol{Q}=\boldsymbol{C}_{2}$. Here, we have, with the same notation as in Theorem $2, K_{1}=\{2\}, K_{2}=\{5\}, K_{3}=\{8\}, K_{4}=$ $\{11\}$. Furthermore, for the function $j$ defined on the set $\{1,2,3,4\}$ by $j(1)=2, j(2)=5, j(3)=8, j(4)=11$, one can verify that any subset $\left\{i_{1}, i_{2}, \ldots, i_{k}\right\}$ of $\{1,2,3,4\}$ is such that $\left(\boldsymbol{C}_{0} \oplus \boldsymbol{C}_{2}\right)_{i_{1}, j\left(i_{1}\right)}+\left(\boldsymbol{C}_{0} \oplus\right.$ $\left.\boldsymbol{C}_{2}\right)_{i_{2}, j\left(i_{2}\right)}+\ldots+\left(\boldsymbol{C}_{0} \oplus \boldsymbol{C}_{2}\right)_{i_{k}, j\left(i_{k}\right)} \geqslant\left(\boldsymbol{C}_{0} \oplus \boldsymbol{C}_{2}\right)_{i_{1}, j\left(i_{2}\right)}+\left(\boldsymbol{C}_{0} \oplus \boldsymbol{C}_{2}\right)_{i_{2}, j\left(i_{3}\right)}+$ $\ldots+\left(\boldsymbol{C}_{0} \oplus \boldsymbol{C}_{2}\right)_{i_{k}, j\left(i_{1}\right)}$. It follows that $\left(\boldsymbol{C}_{0} \oplus \boldsymbol{C}_{2}\right) \boldsymbol{M}=\boldsymbol{C}_{2} \boldsymbol{M}$ admits at least one solution in $\boldsymbol{M}$. Consequently, the existence of a solution for the constraint $\boldsymbol{C}_{0} \boldsymbol{M} \leqslant \boldsymbol{C}_{2} \boldsymbol{M}$ is guaranteed.

- Method 2: direct approach. 
By developping $\boldsymbol{C}_{0} \boldsymbol{M}$ and $\boldsymbol{C}_{2} \boldsymbol{M}$, we have that: $\boldsymbol{C}_{0} \boldsymbol{M} \leqslant \boldsymbol{C}_{2} \boldsymbol{M}$ is equivalent to

$$
\left\{\begin{aligned}
\lambda^{2} \mu^{2} & m_{12, \ell} \leqslant \tau_{1} d_{1} m_{2, \ell} \\
\mu & m_{1, \ell} \leqslant \tau_{2} d_{2} m_{5, \ell} \\
\lambda^{2} \mu^{2} & m_{6, \ell} \leqslant \tau_{1} d_{1} m_{8, \ell} \\
\lambda^{2} \mu^{2} & m_{7, \ell} \leqslant \tau_{2} d_{2} m_{11, \ell}
\end{aligned}\right.
$$

for $\ell=1,2, \ldots, 12$, where $\boldsymbol{M}=\left(m_{i, \ell}\right)_{1 \leqslant i \leqslant 12,1 \leqslant \ell \leqslant 12}$. This system is soluble since the variables involved in every row of Eq. (11) are independent from those of other rows and for a fixed $\ell$, every inequality is of the form $a x \leqslant b y$ with $x$ and $y$ being independant variables.

Now, let us consider the first inequality involved in Eq. (10). The reader can check that $\boldsymbol{C}_{0} \boldsymbol{A}_{0}^{*} \boldsymbol{A}_{1} \leqslant \boldsymbol{C}_{2} \boldsymbol{M}$ reduces to

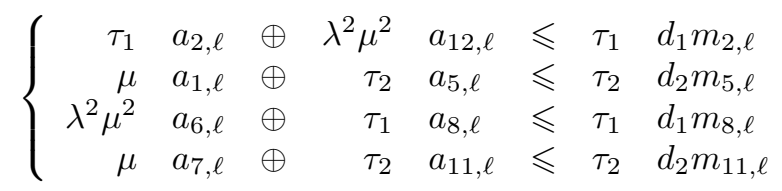

for $\ell=4$ and $\ell=7$, where $\boldsymbol{A}^{\mathrm{ex}}=\boldsymbol{A}_{0}^{*} \boldsymbol{A}_{1}=\left(a_{i, \ell}\right)_{1 \leqslant i \leqslant 12,1 \leqslant \ell \leqslant 12}$.

Finally, in order to solve Eq. (10), we can consider Eqs. (11), (12) and set $m_{12, \ell}=m_{1, \ell}=m_{6, \ell}=m_{7, \ell}=\epsilon$, so that the supervision reduces in solving Eq. (12).

Let us consider a case where no consistant schedule can be achieved without a controller. On one hand, with $\boldsymbol{A}^{\mathrm{ex}}=\boldsymbol{A}_{0}^{*} \boldsymbol{A}_{1}$ calculated for the following numerical values, $\lambda_{0}=14, \lambda=10, \mu=2, \tau_{1}=129, d_{1}=20, \tau_{2}=79, d_{2}=15$, the maximal durations are over not respected, see figure7. On the other hand, the following modification matrix $\boldsymbol{M}^{\mathrm{ex}}$,

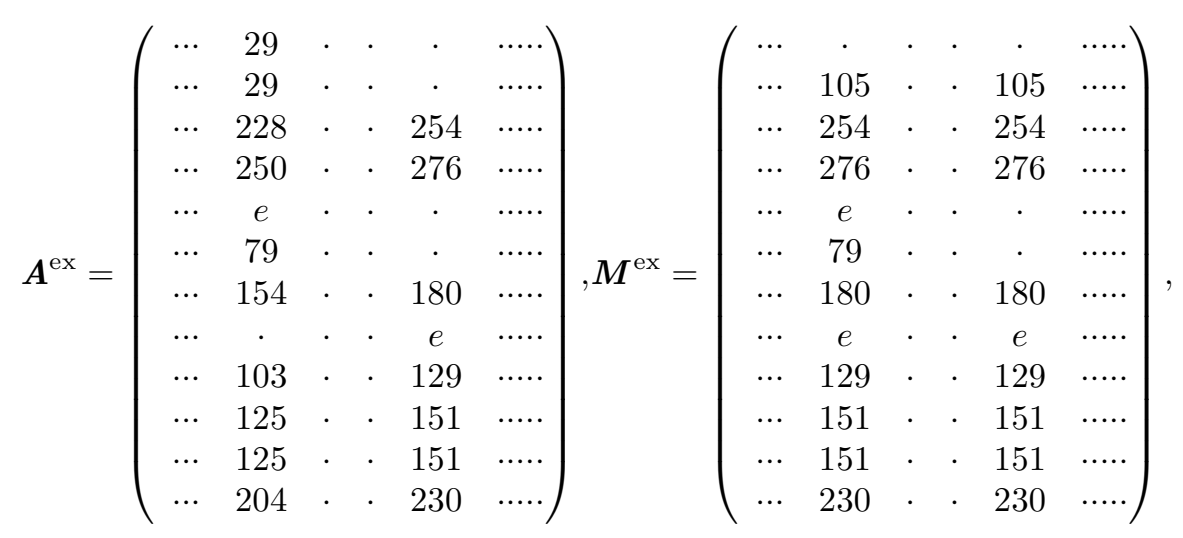

helps respecting all maximal durations constraints.

\section{Application}

A typical example of a system subject to a strict duration constraint is that of a manufacturing unit which includes heat treatment. We are interested in such a manufacturing unit, intended to produce rubber tubes for automobile 


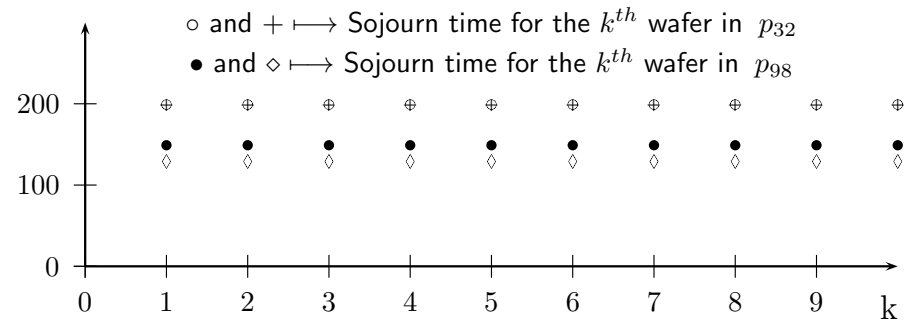

Figure 7: Sojourn time in places $p_{3,2}$, and $p_{9,8}$. Without controller, sojourn time exceeds the maximum admited value $\tau_{1} d_{1}$, while for the modified system, sojourn time in places $p_{3,2}$, and $p_{9,8}$ is always smaller than $\tau_{1} d_{1}$

equipment. The sizing of this industrial plant has been solved and validated via computational simulations in [24], and the resource optimization for the manufacturing unit has been treated in [2]. The description of the industrial manufacturing unit is given in the form of TEG in section 6.1. Then we propose three supervisors which allow of the maximum duration constraint to be respected for the heating zone of the furnace (section 6.2).

\subsection{TEG model for a manufacturing}

The process of interest is an industrial plant specialising in the manufacturing of rubber tubes for automobile equipment. The plant is represented in figure 8. This figure represents three conveyor belts connected in loops. Loops $\mathbf{1}$

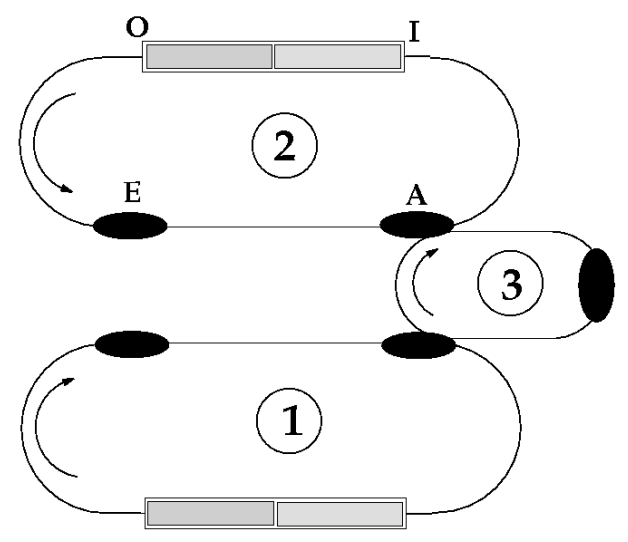

Figure 8: Manufacturing unit.

and $\mathbf{2}$ are identical. Each one is composed of a loading station (A, on loop 2) where parts subject to heat treatment are fixed onto specific pallets, an unloading station (E) where parts are dismounted, and a furnace (IO cells). The furnace itself consists of two zones, a heating zone and a cooling zone. 
The parts are subjected to high temperatures during the time they spent in the first zone of the furnace. Then, they are cooled in the second zone. After cooling of parts, pallets are brought to the unloading station where an operator dismounts the parts and dispatches them in batches towards another unit of the production workshop. The transport device is not always available for the evacuation of treated products and this could cause an accumulation of pallets at the unloading station. In such cases, saturation may occur at the entry of the unloading station, causing a blockage in the system. The pallets present in the furnace then exceed their processing time and the embarked products are burned and lost. Thus, for this application, the time spent in the heating zone is critical: the maximum heating time should not be exceeded even when non-evacuation of treated products occurs at the unloading station.

Loops 1 and $\mathbf{2}$ being identical, we can restrict our attention to one loop (2 in the sequel). This manufacturing unit shows synchronisations between loops. Indeed, loading a pallet is possible only if an empty pallet and parts to be supplied are present at the loading station $\mathbf{A}$. In the same way, availability of the transport device is necessary at the unloading station to take away the treated parts (station $\mathbf{E}$ ) and make available an empty pallet for forthcoming use. This type of industrial plant that uses synchronisations can be modelled as a TEG. The TEG model of this application is that of figure 9. In this graph,

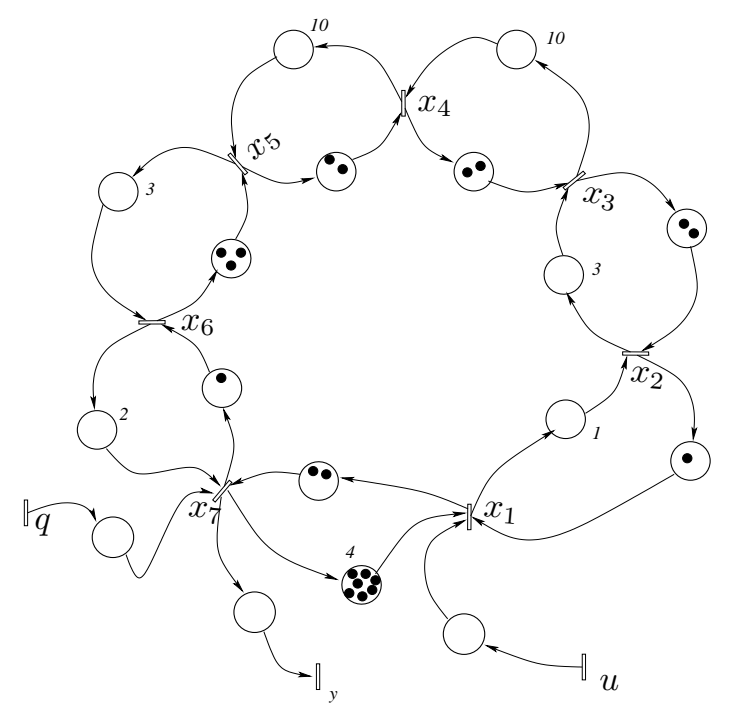

Figure 9: TEG model for the loop $\mathbf{2}$ of the manufacturing unit represented by figure 8 .

transitions are associated with the following events:

- $u$ : arrival of the parts;

- $x_{1}$ : beginning of the loading operation;

- $x_{2}$ : starting transport to the furnace;

- $x_{3}$ : entry to the heating zone of the furnace; 
- $x_{4}$ : entry to the cooling zone of the furnace;

- $x_{5}$ : starting transport to the evacuation zone;

- $x_{6}$ : beginning of the unloading operation;

- $x_{7}$ : part evacuation;

- $q$ : unloading operator (may be present or absent).

- $y$ : departure of the parts.

The input transition $u$ models the arrival of parts to be treated and the transition $q$ models the transport device for evacuating finished parts. When the transport device malfunctions, saturation can occur due to the non-evacuation of treated products. The output transition $y$ corresponds to actually treated and evacuated parts. Crossing transition $x_{i}$ corresponds to the occurrence of an event, for example, crossing $x_{1}$ corresponds to the beginning of the loading operation on a pallet, $x_{2}$ to the end of this operation. Operation durations are indicated close to places; for example, the transfer of a pallet from the loading station to the entry of the furnace (station $\mathbf{I}$ ) is about 3 time units.

Tokens (in places) model the resources of the manufacturing unit: pallets, operators, capacity of conveyors .... For instance, the transfer time from unloading station $\mathbf{E}$ to loading station $\mathbf{A}$ is four time units. In addition, there are actually seven free pallets and there remain two places available on the conveyor.

The state vector, $\boldsymbol{x}$, of this TEG is composed of transitions $x_{1}, x_{2}, \cdots, x_{7}$; and the input vector, $\boldsymbol{v}$, is composed of transitions $u$ and $q$. State and output equations that describe the dynamic behaviour of the TEG of figure 9 are given in $(\max ,+)$-algebra by

$$
\begin{aligned}
& x_{1}(k+1)=x_{2}(k) \quad \oplus 4 x_{7}(k-6) \oplus u(k+1), \\
& x_{2}(k+1)=1 \quad x_{1}(k+1) \oplus x_{3}(k-1), \\
& x_{3}(k+1)=3 \quad x_{2}(k+1) \oplus x_{4}(k-1), \\
& x_{4}(k+1)=10 x_{3}(k+1) \oplus x_{5}(k-1), \\
& x_{5}(k+1)=10 x_{4}(k+1) \oplus x_{6}(k-2), \\
& x_{6}(k+1)=3 \quad x_{5}(k+1) \oplus x_{7}(k), \\
& x_{7}(k+1)=2 x_{6}(k+1) \oplus x_{1}(k-1) \oplus q(k+1), \\
& y(k)=x_{7}(k) .
\end{aligned}
$$

These equations yield a matrix representation where state $\boldsymbol{x}(k)$ at time $k$ depends on states $\boldsymbol{x}(k), \boldsymbol{x}(k-1), \boldsymbol{x}(k-2), \boldsymbol{x}(k-3), \boldsymbol{x}(k-7)$ and on input $\boldsymbol{v}(k)$. However, there exists a simplified representation of the TEG state of the form: $\boldsymbol{x}(k+1)=\boldsymbol{A} \boldsymbol{x}(k) \oplus \boldsymbol{B} \boldsymbol{v}(k+1)$. Indeed, a place with $m$ tokens and temporisation $\alpha$ is equivalent to $m$ places, each of them having only one token and temporisation $\alpha_{i}$, with $\sum \alpha_{i}=\alpha$. According to this decomposition, the TEG model of the manufacturing unit is that of figure 10 (reduction from depth 7 to depth 1).

The dynamic behaviour of the simplified TEG obtained (figure 10) is described by a system of the form:

$$
\left\{\begin{array}{l}
\boldsymbol{x}(k+1)=\boldsymbol{H}_{0} \boldsymbol{x}(k+1) \oplus \boldsymbol{H}_{1} \boldsymbol{x}(k) \oplus \boldsymbol{K}_{0} \boldsymbol{v}(k+1), \\
\boldsymbol{y}(k)=\boldsymbol{S} \boldsymbol{x}(k),
\end{array}\right.
$$




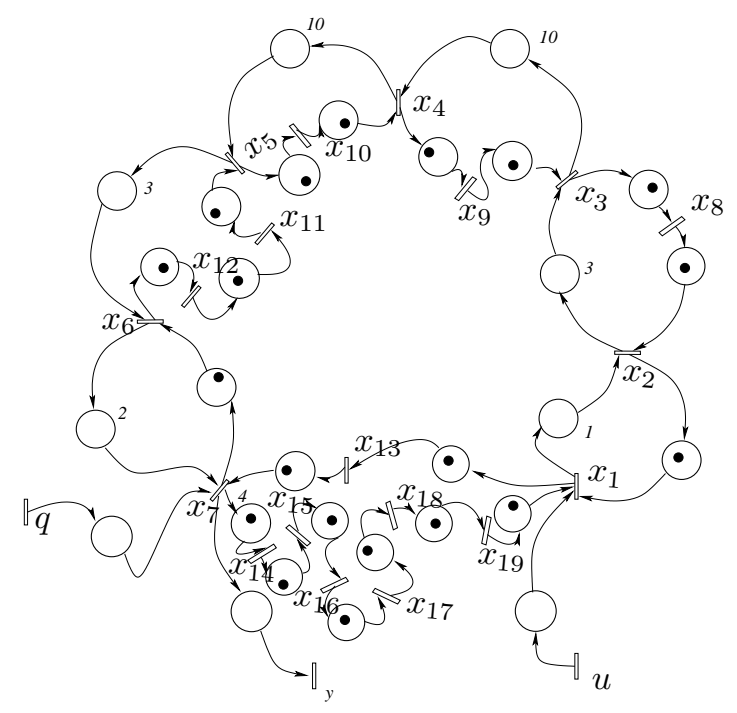

Figure 10: Decomposition of the TEG given in figure 9.

where matrices $\boldsymbol{K}_{0}, \boldsymbol{H}_{0}, \boldsymbol{H}_{1}$ and $\boldsymbol{S}$ are given below. In these matrices, $\epsilon$ has again been replaced by a dot.

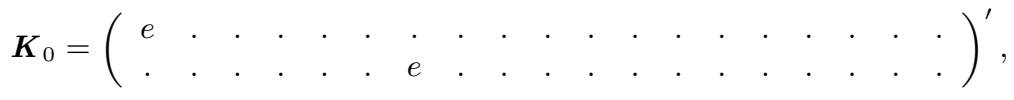

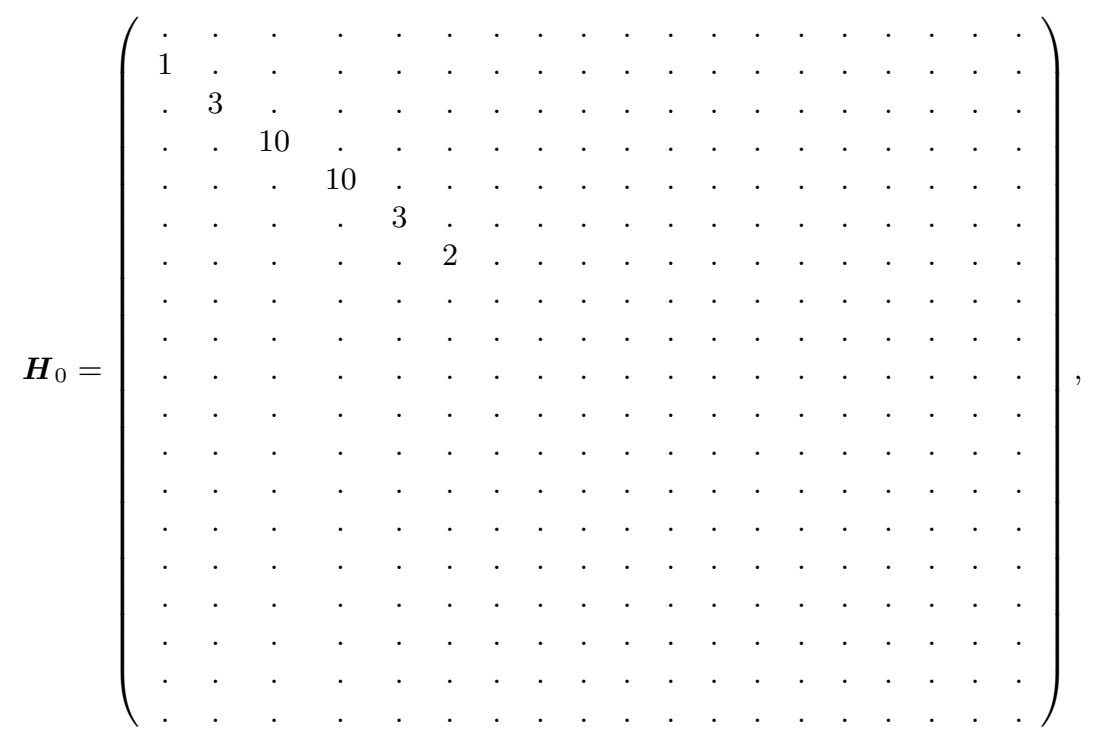




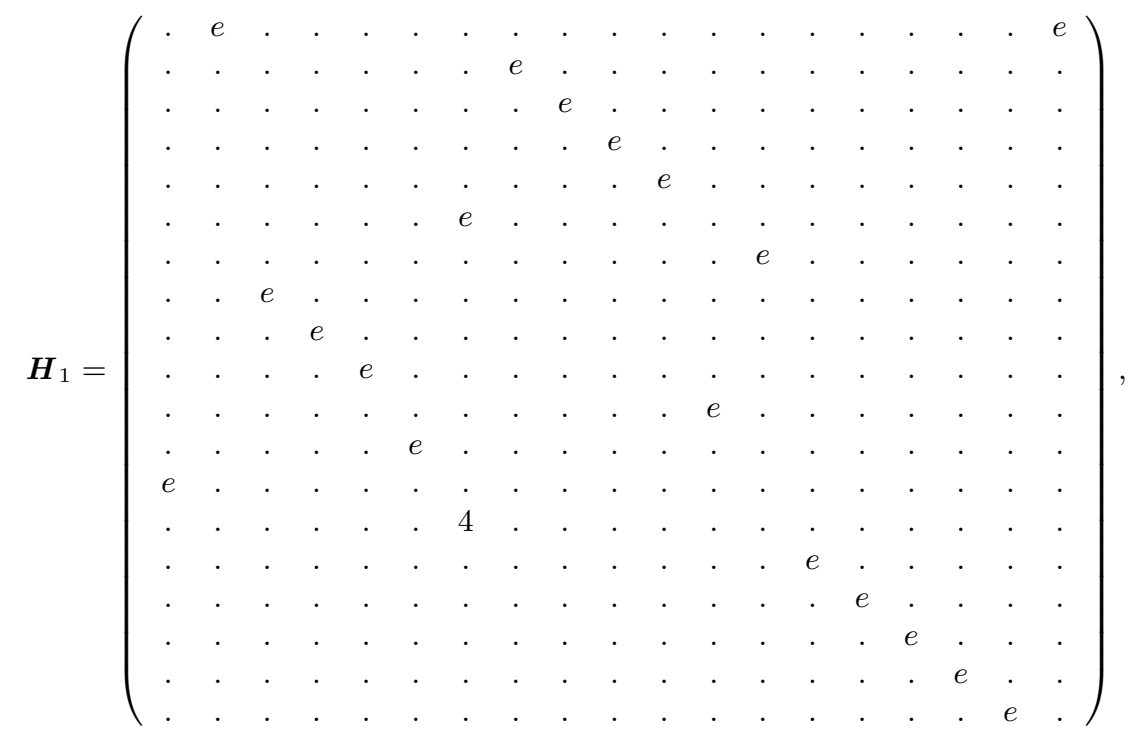

and

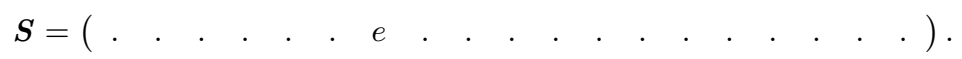

From theorem 1 and the first equation of (14), we are concerned with the system:

$$
\left\{\begin{array}{l}
\boldsymbol{x}(k+1)=\boldsymbol{H}_{0}^{*} \boldsymbol{H}_{1} \boldsymbol{x}(k) \oplus \boldsymbol{H}_{0}^{*} \boldsymbol{K}_{0} \boldsymbol{v}(k+1), \\
\boldsymbol{y}(k)=\boldsymbol{S} \boldsymbol{x}(k) .
\end{array}\right.
$$

Let $\boldsymbol{H}=\boldsymbol{H}_{0}^{*} \boldsymbol{H}_{1}$ and $\boldsymbol{K}=\boldsymbol{H}_{0}^{*} \boldsymbol{K}_{0}$. We thus obtain the following system

$$
\left\{\begin{array}{l}
\boldsymbol{x}(k+1)=\boldsymbol{H} \boldsymbol{x}(k) \oplus \boldsymbol{K} \boldsymbol{v}(k+1), \\
\boldsymbol{y}(k)=\boldsymbol{S} \boldsymbol{x}(k)
\end{array}\right.
$$

which is of the form Eq. (1). Matrices $\boldsymbol{H}_{0}^{*}, \boldsymbol{H}$ and $\boldsymbol{K}$ are given below.

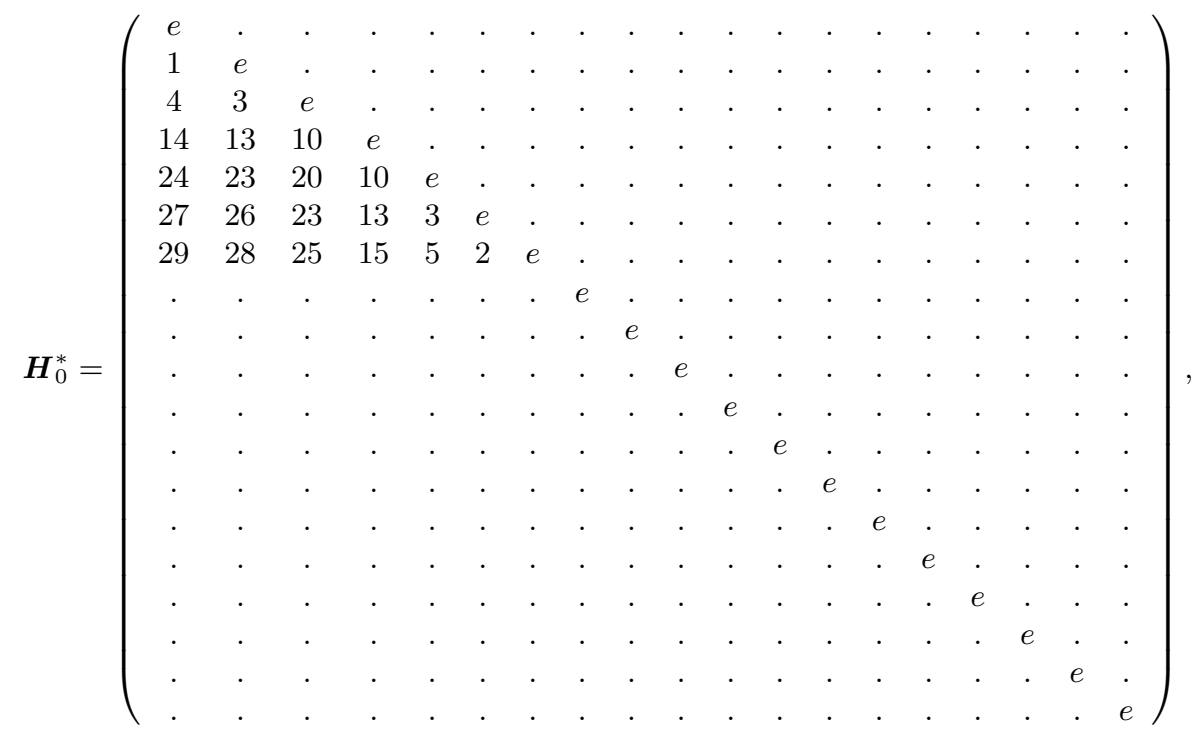




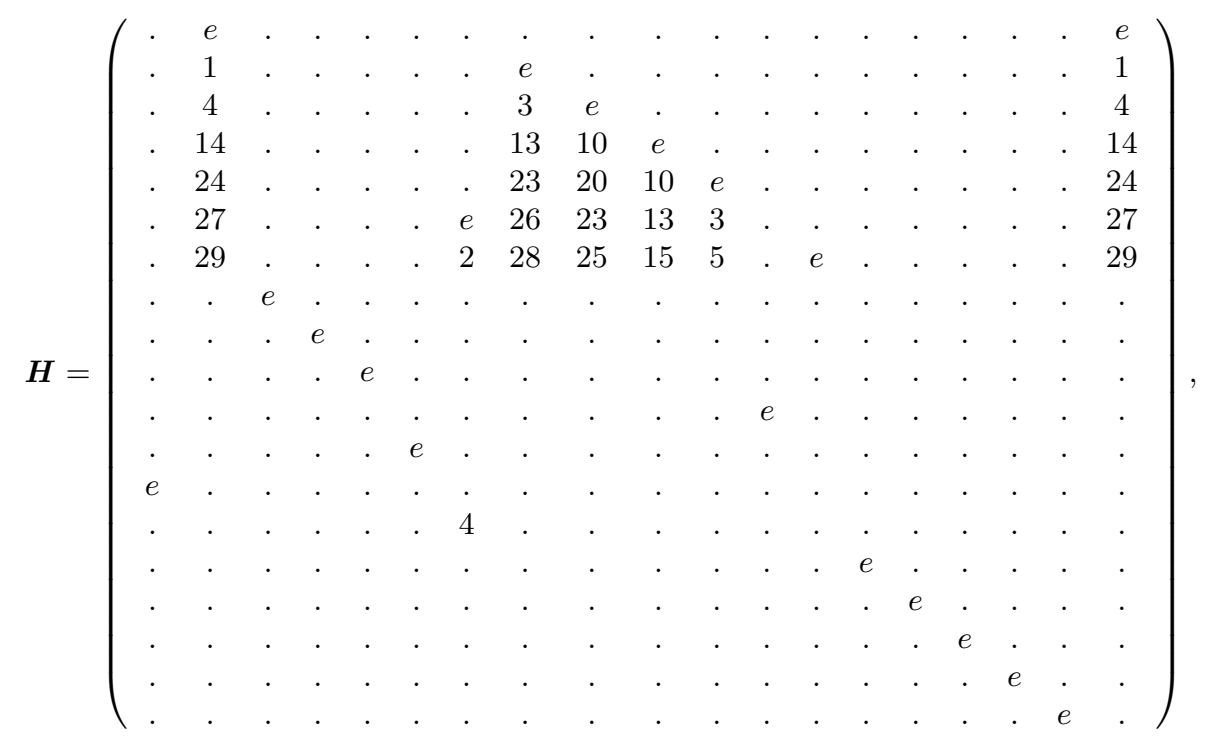

and

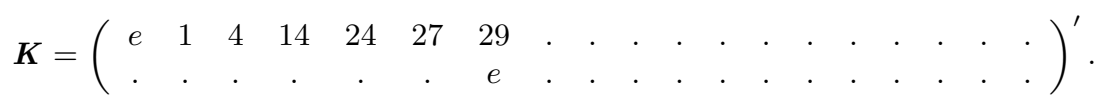

\subsection{Supervision of the industrial plant}

\subsubsection{Constraint expression}

The supervision is aimed at preventing parts being lost because of possible failure in the transport device. The $(\max ,+)$-equation that governs the time spent by a part in the heating zone of the furnace is derived from the dynamic behaviour of the simplified TEG of figure 10 and is:

$$
x_{4}(k+1)=10 x_{3}(k+1) \oplus x_{10}(k) .
$$

To avoid losing parts, a product should not exceed 10 time units in the heating zone of the furnace (place that links transition $x_{3}$ to transition $x_{4}$ in figures 9 and 10). Thus, this constraint will be respected by forcing

$$
x_{4}(k+1) \leqslant 10 x_{3}(k+1),
$$

Taking Eq. (17) into account, condition Eq. (18) will be satisfied iff:

$$
x_{10}(k) \leqslant 10 x_{3}(k+1) \text {. }
$$

Denoting

$$
\boldsymbol{Q}_{1}=(. . . \quad . \quad . \quad . \quad . \quad . \quad . \quad e \quad . \quad . \quad . \quad . \quad . \quad . \quad . \quad . \quad .),
$$

and

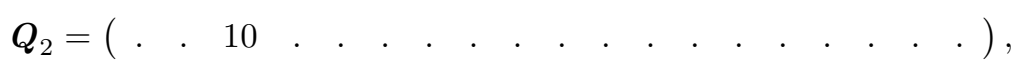

the constraint condition Eq. (19) is thus of the form Eq. (7): $\boldsymbol{Q}_{1} \boldsymbol{x}(k) \leqslant$ $\boldsymbol{Q}_{2} \boldsymbol{x}(k+1)$. 


\subsubsection{Supervision of the manufacturing unit}

The dynamic behaviour of the manufacturing unit is described with the $(\max ,+)$ system Eq. (16). The maximum duration constraint imposes

$$
\boldsymbol{Q}_{1} \boldsymbol{x}(k) \leqslant \boldsymbol{Q}_{2} \boldsymbol{x}(k+1) .
$$

From theorem 3, a supervisor guaranteeing respect of the duration constraint can be calculated by applying a state modification of the form

$$
\boldsymbol{x}(k+1)=\left(\boldsymbol{H} \oplus \boldsymbol{M}_{0}\right) \boldsymbol{x}(k) \oplus \boldsymbol{K} \boldsymbol{v}(k+1),
$$

where $M_{0}$ (supervision matrix) is a matrix satisfying

$$
Q_{1} \leqslant Q_{2} M_{0}
$$

The only non null element of $\boldsymbol{Q}_{1}$ being $\left(\boldsymbol{Q}_{1}\right)_{1,10}=e$, it is sufficient to consider the solutions of:

$$
e \leqslant \bigoplus_{j=1}^{19}\left(\boldsymbol{Q}_{2}\right)_{1, j}\left(\boldsymbol{M}_{0}\right)_{j, 10},
$$

that is,

$$
e \leqslant 10\left(\boldsymbol{M}_{0}\right)_{3,10} .
$$

The smallest positive solution of the latter equation is $\left(\boldsymbol{M}_{0}\right)_{3,10}=e$. The supervision obtained from this solution involves adding to the graph of figure 10, a place having a single token (with no temporisation because $\left(\boldsymbol{M}_{0}\right)_{3,10}=e \equiv 0$ ) from transition $x_{10}$ to transition $x_{3}$.

Let ${ }_{m} x_{3}$ be the state of transition $x_{3}$ after supervision. The firing of transition $m_{3} x_{3}$ is then subject to

$$
{ }_{m} x_{3}(k+1)=x_{3}(k+1) \oplus x_{10}(k) .
$$

Transition $x_{10}$ being an auxiliary variable derived from the expansion of the original model of the manufacturing unit (represented by the graph of figure 9), we do not have access to this transition in practice: it is neither controllable, nor observable [12]. But from Eq. (16) we derive that $x_{10}(k)=x_{5}(k-1)$, and equation Eq. (24) becomes

$$
{ }_{m} x_{3}(k+1)=x_{3}(k+1) \oplus x_{5}(k-1) .
$$

The resulting graph modification involves adding a single place with two tokens from transition $x_{5}$ to transition $x_{3}$. State modification driven by Eq. (25) leads to the supervisor represented in figure 11. This supervision involves imposing only two tokens in the cycle $x_{3} \rightarrow x_{5} \rightarrow x_{3}$ which corresponds to IO cells of the plant (figure 8 ), that is, the whole furnace (heating and cooling zone). It is easy to check that if we do this, no product will remain more than 10 time units in the heating zone of the furnace because there will be a free place in the cooling zone. This supervision guarantees that parts (a maximum of two parts) entering the furnace cannot be lost even when saturation occurs at the evacuation station.

In a similar way, we can obtain other supervisors from Eq. (25), and by taking into account other equations of Eq. (13) (for controllability reasons). 


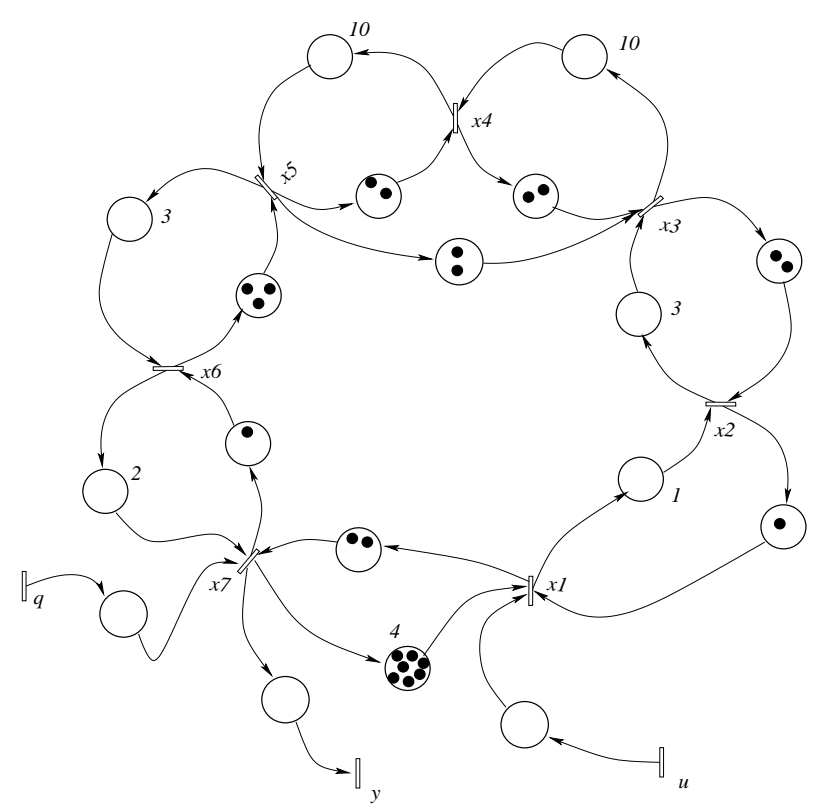

Figure 11: First supervision.

Indeed, from the fifth equation of Eq. (13), we have $x_{5}(k-1)=10 x_{4}(k-1) \oplus$ $x_{6}(k-4)$. In this equation, $10 x_{4}(k-1)$ represents the normal incrementation of the process due to the dynamic behaviour of the TEG, and $x_{6}(k-4)$ represents availability of a resource (conveying to the unloading station). Non-evacuation of treated parts affects the availability of the resource: there is no more place at the unloading station to receive new parts and the treated parts accumulate in the conveyor. It follows that $x_{5}(k-1)=x_{6}(k-4)$. From this latter equation and Eq. (25), we thus obtain the state modification:

$$
{ }_{m} x_{3}(k+1)=x_{3}(k+1) \oplus x_{6}(k-4) .
$$

This new supervision involves adding a single place with five tokens from transition $x_{6}$ to transition $x_{3}$. This leads to the supervision presented in figure 12 . The supervisor imposes a maximum of five tokens in cycle $x_{3} \rightarrow x_{6} \rightarrow x_{3}$. It is also easy to see that imposing five tokens in this cycle makes it possible to ensure that no parts will remain more than 10 time units in the heating zone of the furnace: five place are available in the cycle $x_{4} \rightarrow x_{6} \rightarrow x_{4}$. This supervision is represented in figure 12.

Finally, and in a similar way, a third supervisor is calculated from Eq. (26) and the sixth equation of Eq. (13). Indeed, we have $x_{6}(k-4)=3 x_{5}(k-4) \oplus$ $x_{7}(k-5)$, where $3 x_{5}(k-4)$ represents the normal incrementation of a process due to the dynamic behaviour of the TEG and $x_{7}(k-5)$ represents availability of a resource (unloading operator). Non-evacuation of treated parts only affects resource availability and it follows that $x_{6}(k-4)=x_{7}(k-5)$. We thus obtain from this equation and Eq. (26) the corresponding state modification:

$$
{ }_{m} x_{3}(k+1)=x_{3}(k+1) \oplus x_{7}(k-5) .
$$




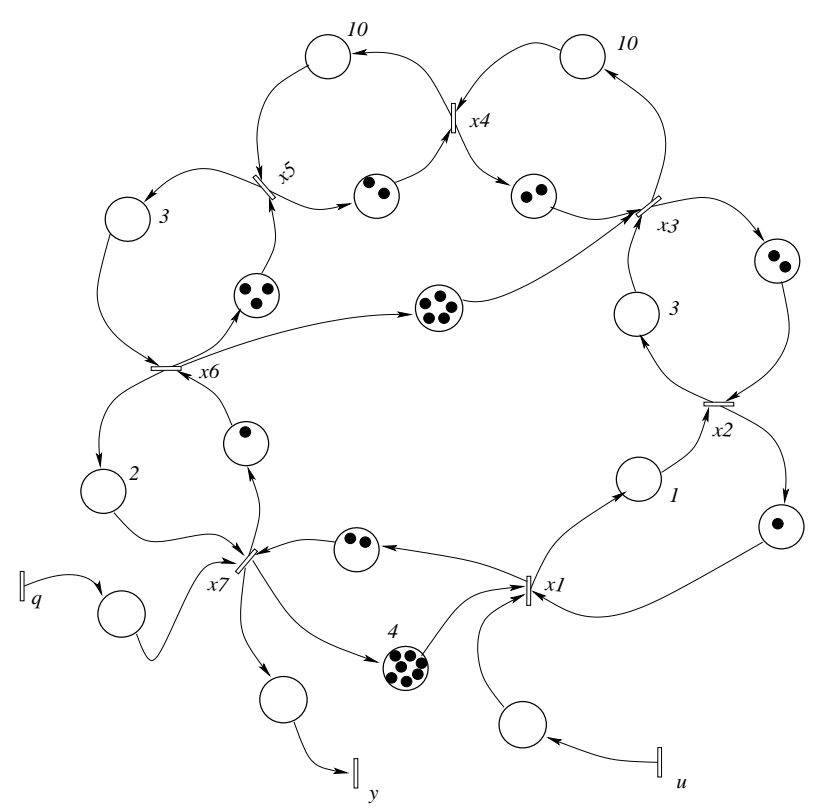

Figure 12: Second supervision.

By proceeding in this way, the supervision involves imposing six tokens in cycle $x_{3} \rightarrow x_{7} \rightarrow x_{3}$. The supervision obtained is given in figure 13 .

\subsubsection{Classification of supervisors}

This section discusses the classification of supervisors synthesised in section 6.2.2. Classification is addressed by comparing the production rate yielded by "TEG+supervisor" systems, in comparison with the production rate of the non-supervised manufacturing unit. For this purpose, we compute cycle times associated with the TEGs of figures 9, 11, 12 and 13. The cycle time is the inverse of the production throughput.

Let $\lambda$ be the cycle time of the non-supervised manufacturing unit (figure 9). The following procedure (see $[9,19])$ makes it easy to determine $\lambda$ : for every cycle $i$ (sequence of vertices and arcs which allows a direct connection from $i$ to i) of the graph, determine the ratio

$$
\lambda_{i}=\frac{\text { Sum of cycle temporisations }}{\text { Number of tokens in the cycle }} .
$$

Then, $\lambda$ is the maximum of $\lambda_{i}$.

From the above procedure, the cycle time of the non-supervised unit is:

$$
\lambda=\max \left\{\frac{33}{7}, \frac{1}{1}, \frac{3}{2}, \frac{10}{2}, \frac{10}{2}, \frac{3}{3}, \frac{2}{1}, \frac{0}{13}\right\}=5,
$$

and the cycle times $\lambda^{\prime}, \lambda^{\prime \prime}$ and $\lambda^{\prime \prime \prime}$ of the unit supervised according to figures 11,12 and 13 respectively, are:

$$
\lambda^{\prime}=\max \left\{\lambda, \frac{20}{2}\right\}=10
$$




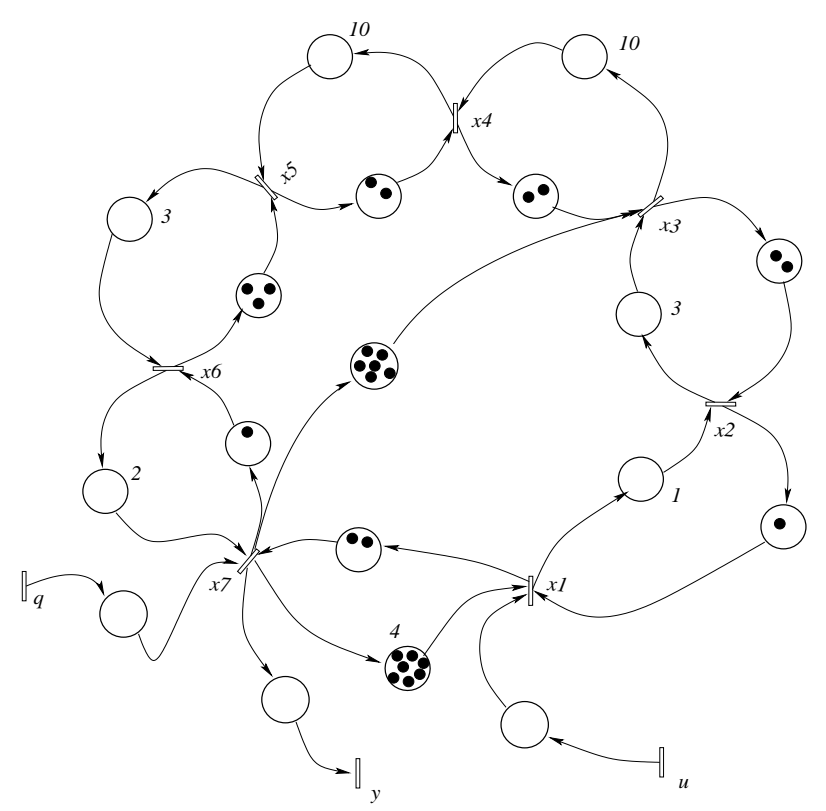

Figure 13: Third supervision.

$$
\begin{aligned}
& \lambda^{\prime \prime}=\max \left\{\lambda, \frac{23}{5}\right\}=5, \\
& \lambda^{\prime \prime \prime}=\max \left\{\lambda, \frac{25}{6}\right\}=5 .
\end{aligned}
$$

The cycle time $\lambda^{\prime}$ of the supervised TEG of figure 11 is greater than $\lambda$. Thus, the supervision represented in figure 11 affects the production throughput of the industrial plant. In contrast, $\lambda^{\prime \prime}=\lambda^{\prime \prime \prime}=\lambda$ : supervisors represented in figures 12 and 13 preserve the initial production throughput of the industrial plant. Now, between the TEGs in figures 12 and 13, the last one has the smallest supervisor's cycle time (cycle time yielded by adding the supervisor's place) since $23 / 5$ is greater than $25 / 6$. As a matter of fact, both supervisors lead to the same resulting throughput for the plant and they are therefore equivalent according to production throughput criteria.

To illustrate the plant functioning with and without supervision, assume that the unloading operator may not be present unless there are 100 time units. If input $u$ is free of control, then figure 14 displays firing of transitions $u, x_{3}, x_{4}$, and $y$ for the non-supervised plant and figure 15 shows the firing times of these transitions for the supervised graph given by figure 13 . We observe that the delay of firing between $x_{3}(6)$ and $x_{4}(6)$ is 86 for the non-supervised plant: products are lost, while supervision ensures respect of the time constraint which is 10 time units in the place that links transitions $x_{3}(k)$ to $x_{4}(k)$.

\section{Conclusion}

The work presented in this paper tackles the problem of the synthesis of supervisors aimed at guaranteeing maximum duration constraints in TEGs. Results 


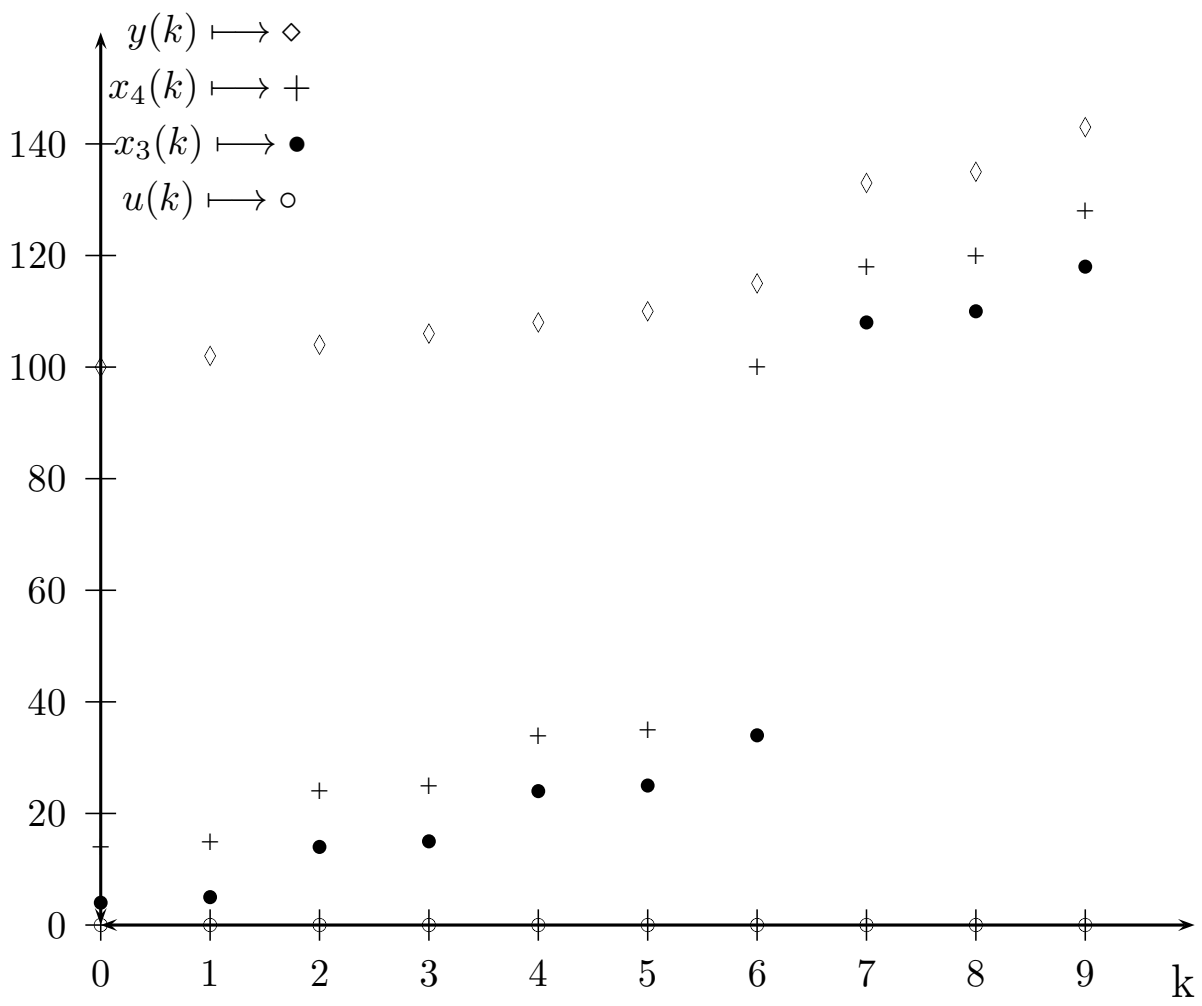

Figure 14: Unsupervised graph.

are given by considering solutions of constrained state equations. The approach proposed leads to the derivation of a whole class of supervisors. A classification method is then needed to evaluate their performance. In the application treated, we propose the cycle time as the performance criterion. We observe that two supervisors obtained from our method preserve the original cycle time of the production unit, that is, they impose respect of the maximum duration constraint without impacting the production throughput of the industrial unit.

The approach used in this work can be extended to other cases involving more stringent constraints and this can probably lead to less direct synthesis of supervisors. In future work, we plan to investigate the interesting problem of being able to directly integrate performance parameters into the constraint expression, in order that the derived supervisors do not affect the production throughput of the original system.

\section{References}

[1] X. Allamigeon, S. Gaubert, and E. Goubault. Algorithmics of tropical polyhedra. submitted, 2010.

[2] S. Amari, I. Demongodin, J.J. Loiseau, and C. Martinez. Sizing and cycle time of an industrial plant using dioid algebra. In $9^{\text {th }}$ International Multi- 


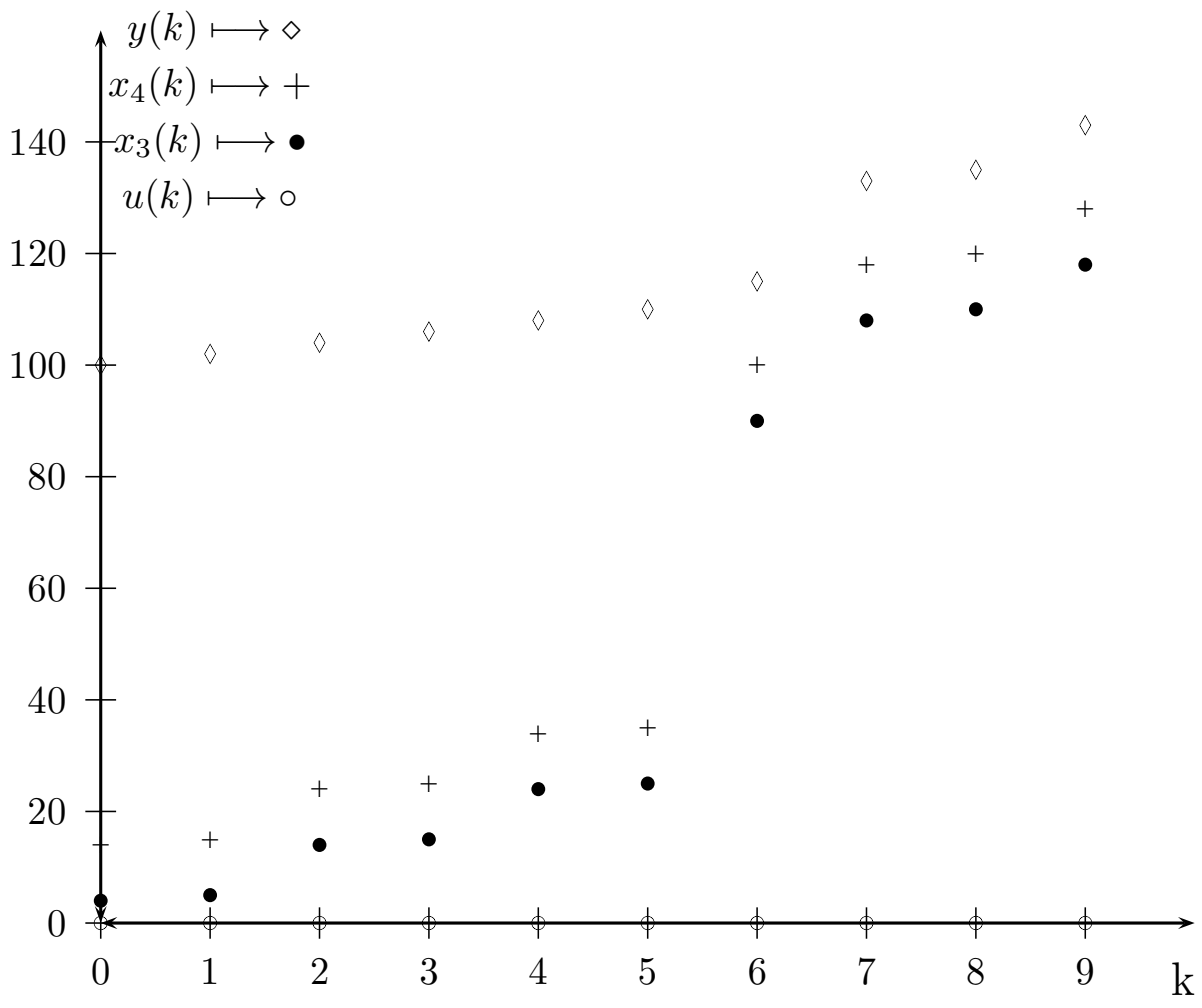

Figure 15: Supervised graph.

conference on Advanced Computer Systems - Production System Design Supply Chain Management (ACS'02-SCM), pages pp 99-106, 2002.

[3] S. Amari, J.J. Loiseau, and I. Demongodin. Control of linear min-plus systems under temporal constraints. In IEEE Conference on Decision and Control and the European Control Conference, Seville, Spain, pages pp 7738-7743, 2005.

[4] F. Baccelli, G. Cohen, G.J. Olsder, and J.P. Quadrat. Synchronization and Linearity. Wiley, 1992.

[5] P. Butkovič, H. Schneider, and S. Sergeev. Generators, extremals and bases of max cones. Linear Algebra and its Applications, 421(2-3):394-406, 2007.

[6] C.G. Cassandras and S. Lafortune. Introduction to discrete event systems. Kluwer Academic, 1992.

[7] K. Cechlárová. Eigenvectors of interval matrices over max-plus algebra. Discrete Applied Mathematics, 150:pp 2-15, 2005.

[8] G. Cohen. Dioids and discrete event systems. In Proc. of the 11th Conf. on Anal. and Opt. of Systems: Discrete Event Systems, editors, Lect. Notes. in Control and Inf. Sci, number 199. Springer, Sophia Antipolis, 1994. 
[9] G. Cohen, D. Dubois, J.P. Quadrat, and M. Viot. Analyse du comportement périodique des systèmes de production par la théorie des diö̈des. Technical Report 191, INRIA, 1983.

[10] G. Cohen, D. Dubois, J.P. Quadrat, and M. Viot. A linear system theoretic view of discrete event processes and its use for performance evaluation in manufacturing. IEEE Trans. on Automatic Control, 30:pp 210-220, 1985.

[11] G. Cohen, P. Moller, J.P. Quadrat, and M. Viot. Algebraic tools for the performance evaluation of discrete event systems. 1985.

[12] C. Commault. Feedback stabilisation of some event graph models. IEEE Trans. on Automatic Control, 1998.

[13] R. A. Cuninghame-Green and P. Butkovič. The equation $a \otimes x=b \otimes y$ over (max, +). Theor. Comput. Sci., 293(1):3-12, 2003.

[14] S. Gaubert. Théorie des systémes linéaires dans les diö̈des. PhD thesis, École des Mines de Paris, 1992.

[15] S. Gaubert. Resource optimization and (min,+) spectral theory. IEEE Trans. on Automatic Control, 40(11):pp 1931-1934, 1995.

[16] M. Gondran and M. Minoux. Valeurs propres et vecteurs propres dans les dioïdes et leur interprétation en théorie des graphes. Technical Report 2, EDF, Bulletin de la Direction des Etudes et Recherches, Serie C, Mathématiques Informatique, 1977.

[17] G. Hegedüs and P. Butkovič. An elimination method for finding all solutions of the system of linear equations over an extremal algebra. Ekonomickomatematicky Obzor, 20:203-215, 1984.

[18] B. Heidergott, G.J. Olsder, and J; van der Woude. Max Plus at Work: Modeling and Analysis of Synchronised Systems. Princeton and Oxford, 2006.

[19] H.P. Hillion and J. Proth. Performance evaluation of job-shopsystems using timed event-graphs. IEEE Trans. on Automatic Control, 34(1):3-9, 1989.

[20] L. Houssin, S. Lahaye, and J.L. Boimond. Just in time control of constrained (max, +)-linear systems. Journal of Discrete Event Dynamic Systems, 17:pp 159-178, 2007.

[21] J.H. Kim and T.E. Lee. Schedule stabilization and robust timing control for time-constrained cluster tools. In IEEE International Conference on Robotics and Automation, Taipei, Taiwan, pages pp 1039-1044, 2003.

[22] A.M. Law and W.D. Kelton. Simulation Modeling and Analysis. McGrawHill, New York, 1991.

[23] L. Libeaut. Sur l'utilisation des diö̈des pour la commande des systèmes à événements discrets. PhD thesis, ECN - Université de Nantes, 1996. 
[24] C. Martinez and P. Castagna. Sizing of an industrial plant using tight time constraints using complementary approaches: $(\max ,+)$ theory and computer simulation. Elsevier, Simulation Practice and Theory, 11:pp 75$88,2003$.

[25] T. Murata. Petri nets: Properties, analysis and applications. IEEE Proceedings, 77(4):pp 541-580, 1989.

[26] I. Ouerghi and L. Hardouin. Control synthesis for p-temporal event graphs. In WODES'06, Ann Arbor University of Michigan, USA, 2006.

[27] P. Spacek, M.A. Manier, and A.E. Moudni. Control of an electroplating line in the max and min algebras. International Journal of Systems Science, 30(7):pp 759-778, 1999.

[28] E. Walkup. Optimization of Linear Max-Plus Systems with Application to Timing Analysis. PhD thesis, University of Washington, 1995.

[29] N. Wu, C. Chu, F. Chu, and M.C. Zhou. A petri net method for schedulability and scheduling problems in single-arm cluster tools with wafer residency time constraints. IEEE Transactions on Semiconductor Manufacturing, 21(2):224-237, 2008. 